\title{
Calculation of Optical Parameters of Liquid Crystals
}

\author{
A. KumaR* \\ Department of Physics, Vardhman College Bijnor \\ Bijnor-246701, UP, India
}

(Received July 16, 2007; in final form November 5, 2007)

\begin{abstract}
Validation of a modified four-parameter model describing temperature effect on liquid crystal refractive indices is being reported in the present article. This model is based upon the Vuks equation. Experimental data of ordinary and extraordinary refractive indices for two liquid crystal samples MLC-9200-000 and MLC-6608 are used to validate the above-mentioned theoretical model. Using these experimental data, birefringence, order parameter, normalized polarizabilities, and the temperature gradient of refractive indices are determined. Two methods: directly using birefringence measurements and using Haller's extrapolation procedure are adopted for the determination of order parameter. Both approches of order parameter calculation are compared. The temperature dependences of all these parameters are discussed. A close agreement between theory and experiment is obtained.
\end{abstract}

PACS numbers: 78.20.-e, 78.20.Ci, 78.20.Fm, 77.84.Nh

\section{Introduction}

Liquid crystals (LCs) are important for fundamental research and for their applicability in display technology. Liquid crystals are best known for their application in displays; besides it they are widely used in liquid crystal thermometers [1], tunable microlenses [2], light modulators [3], switchable devices [4], light shutters [5], and many more.

The refractive indices of a liquid crystal are mainly determined by molecular structure, operating wavelength, and temperature. Various theoretical models [6-9] have been developed to establish the wavelength and temperature dependence of LC refractive indices. In our earlier work we have developed a modified four-parameter model [10] which describes the temperature dependence of ordinary refractive index $\left(n_{\mathrm{o}}\right)$ and extraordinary refractive index $\left(n_{\mathrm{e}}\right)$. The validation of this model is made here by fitting the theoretical values with experimental data of refractive indices. In order to validate this theoretical model, the refractive

*e-mail: anantphy@gmail.com 
indices $\left(n_{\mathrm{e}}, n_{\mathrm{o}}\right)$ data of two liquid crystal samples MLC-9200-000 and MLC-6608 have been used. Refractive indices measurements of these liquid crystal samples were carried out in the temperature range of $288-328 \mathrm{~K}$ by $\mathrm{Li}$ et al. [11] at a wavelength of $\lambda=589 \mathrm{~nm}$. Using the refractive indices data, it is possible to determine some other relevant parameters such as birefringence $(\Delta n)$, order parameter $(Q, S)$, normalized polarizabilities $\left(\alpha_{\mathrm{e}} /\langle\alpha\rangle, \alpha_{\mathrm{o}} /\langle\alpha\rangle\right)$, and the temperature gradient of refractive indices $\left(\mathrm{d} n_{\mathrm{e}} / \mathrm{d} T, \mathrm{~d} n_{\mathrm{o}} / \mathrm{d} T\right)$.

The order parameter indicates the percent of the mesogenic molecules aligned in parallel with the director. Two procedures are discussed for the determination of order parameter. The first one is a simple procedure solely based on birefringence measurements [12]. This procedure consists in the following steps: measurements of birefringence $\Delta n$, determination of $\Delta n_{\mathrm{o}}$ using extrapolation, and calculation of order parameter. The second procedure employed for the determination of order parameter is Haller's extrapolation method [13]. In this method the well-known relation given by Vuks is used [14]. Temperature dependent experimental results of all these parameters are linked with the theoretical value. It turns out that the relationship between the theoretical values and experimental data agrees fairly well.

\section{Theory}

\subsection{Modified four-parameter model}

For an anisotropic liquid crystal there are two principal refractive indices, $n_{\mathrm{e}}$ and $n_{\mathrm{o}}$ for the extraordinary and ordinary rays, respectively. Vuks made a bold assumption that the internal field in a crystal is the same in all directions. With this assumption, he derived the following equation for anisotropic medium [14] which relates ordinary and extraordinary refractive indices to the corresponding polarizabilities:

$$
\frac{n_{\mathrm{e}, \mathrm{o}}^{2}-1}{\left\langle n^{2}\right\rangle+2}=\frac{4}{3} N \pi \alpha_{\mathrm{e}, \mathrm{o}},
$$

where $\alpha_{\mathrm{e}}$ and $\alpha_{\mathrm{o}}$ are molecular polarizabilities, $N$ is a number of molecules in a volume unit and $\left\langle n^{2}\right\rangle$ is the mean value of the square of refractive index given by the following equation:

$$
\left\langle n^{2}\right\rangle=\frac{n_{\mathrm{e}}^{2}+2 n_{\mathrm{o}}^{2}}{3} .
$$

Based on this equation, earlier we have presented the following expressions [10] for ordinary and extraordinary refractive indices as a function of $\sqrt{\left\langle n^{2}\right\rangle}$ and the birefringence $\left(\Delta n=n_{\mathrm{e}}-n_{\mathrm{o}}\right)$ :

$$
\begin{aligned}
& n_{\mathrm{e}}=\sqrt{\left\langle n^{2}\right\rangle}+\frac{2}{3} \Delta n, \\
& n_{\mathrm{O}}=\sqrt{\left\langle n^{2}\right\rangle}-\frac{1}{3} \Delta n,
\end{aligned}
$$

To describe the temperature dependence of birefringence, Haller's approximation [13] is commonly used 


$$
\Delta n=\Delta n_{\mathrm{o}}\left(1-\frac{T}{T_{\mathrm{c}}}\right)^{\beta} .
$$

In Eq. (4) $\Delta n_{\mathrm{o}}$ is the LC birefringence in the crystalline state (or at $T=0 \mathrm{~K}$ ), $\beta$ is a material constant, and $T_{\mathrm{c}}$ is the clearing temperature of LC material under investigation. Using the experimental data of $n_{\mathrm{e}}$ and $n_{\mathrm{o}}$, we find that $\sqrt{\left\langle n^{2}\right\rangle}$ decreases linearly with temperature, so it can be written as

$$
\sqrt{\left\langle n^{2}\right\rangle}=A+B T \text {. }
$$

Substituting values of $\Delta n$ and $\sqrt{\left\langle n^{2}\right\rangle}$ from Eqs. (4) and (5) in Eqs. (3A) and (3B), the modified four-parameter model [10] is obtained. This model describes the temperature dependence of $\mathrm{LC}$ refractive indices

$$
\begin{aligned}
& n_{\mathrm{e}}=A+B T+\frac{2}{3} \Delta n_{\mathrm{o}}\left(1-\frac{T}{T_{\mathrm{c}}}\right)^{\beta}, \\
& n_{\mathrm{o}}=A+B T-\frac{1}{3} \Delta n_{\mathrm{o}}\left(1-\frac{T}{T_{\mathrm{c}}}\right)^{\beta},
\end{aligned}
$$

Equation (6) contains four unknown parameters. These four parameters can be easily obtained by fitting the experimental data of $\sqrt{\left\langle n^{2}\right\rangle}$ and $\Delta n$. A fitting curve of temperature dependent $\sqrt{\left\langle n^{2}\right\rangle}$ using Eq. (5), gives the value of $A$ and $B . \Delta n_{\mathrm{o}}$ and $\beta$ are obtained by fitting the experimental data for $\Delta n$ to Eq. (4) written in a logarithmic form

$$
\log \Delta n=\log \Delta n_{\mathrm{o}}+\beta \log \left(1-\frac{T}{T_{\mathrm{c}}}\right) .
$$

\subsection{Determination of order parameter}

The first approach of the order parameter determination uses Haller's approximation, according to which the order parameter can be approximated as $Q=\left(1-T / T_{\mathrm{c}}\right)^{\beta}$. Using this equation with Eq. (4), Eq. (3) can be written as

$$
\begin{aligned}
& n_{\mathrm{e}}=\sqrt{\left\langle n^{2}\right\rangle}+\frac{2}{3} \Delta n_{\mathrm{o}} Q, \\
& n_{\mathrm{o}}=\sqrt{\left\langle n^{2}\right\rangle}-\frac{1}{3} \Delta n_{\mathrm{o}} Q .
\end{aligned}
$$

After subtraction of Eqs. (8A) and (8B), we find the following relation for the order parameter $(Q)$ :

$$
Q=\frac{\Delta n}{\Delta n_{\mathrm{o}}}
$$

This method is very simple and can be directly applied to the birefringence data rather than to refractive indices.

In the second approach, the orientational order parameter is calculated using the Vuks hypothesis [14]:

$$
S\left(\frac{\Delta \alpha}{\alpha}\right)=\frac{n_{\mathrm{e}}^{2}-n_{\mathrm{o}}^{2}}{\left\langle n^{2}\right\rangle-1}
$$

where $\Delta \alpha$ denotes the anisotropy of molecular polarizability $\alpha$. 
In order to determine the order parameter $(S)$, Haller's extrapolation method is followed. In this method, the term $\log \left(\frac{n_{\mathrm{e}}^{2}-n_{\mathrm{o}}^{2}}{\left\langle n^{2}\right\rangle-1}\right)$ has been plotted as a function of $\log \left(1-T / T_{\mathrm{c}}\right)$. This plot comes out to be linear and can be extrapolated to $T=0 \mathrm{~K}$. The intercept at $T=0 \mathrm{~K}$ where the complete ordering occurs (i.e. $S=1$ ), gives the value of $\Delta \alpha / \alpha$. Substituting this value in Eq. (10), we find the required order parameter. The above-mentioned second procedure is used very traditionally for the evaluation of order parameter .

\subsection{Normalized polarizabilities and temperature gradient of refractive indices}

The normalized polarizabilities for extraordinary and ordinary rays are given by the following equations:

$$
\frac{\alpha_{\mathrm{e}, \mathrm{o}}}{\langle\alpha\rangle}=\frac{n_{\mathrm{e}, \mathrm{o}}^{2}-1}{\left\langle n^{2}\right\rangle-1}
$$

where $\langle\alpha\rangle$ is the mean polarizability given by $\langle\alpha\rangle=\left(\alpha_{\mathrm{e}}+2 \alpha_{\mathrm{o}}\right) / 3$. Ratio of normalized polarizabilities can be expressed as

$$
\frac{\alpha_{\mathrm{e}}}{\alpha_{\mathrm{o}}}=\frac{n_{\mathrm{e}}^{2}-1}{n_{\mathrm{o}}^{2}-1}
$$

The temperature gradients for liquid crystal refractive indices $\left(n_{\mathrm{e}}, n_{\mathrm{o}}\right)$ can be found out from Eqs. (6A) and (6B):

$$
\begin{aligned}
& \frac{\mathrm{d} n_{\mathrm{e}}}{\mathrm{d} T}=B-\frac{2 \beta \Delta n_{\mathrm{o}}}{3 T_{\mathrm{c}}}\left(1-\frac{T}{T_{\mathrm{c}}}\right)^{\beta-1}, \\
& \frac{\mathrm{d} n_{\mathrm{o}}}{\mathrm{d} T}=B+\frac{\beta \Delta n_{\mathrm{o}}}{3 T_{\mathrm{c}}}\left(1-\frac{T}{T_{\mathrm{c}}}\right)^{\beta-1} .
\end{aligned}
$$

At a critical temperature, $\mathrm{d} n_{\mathrm{o}} / d T$ jumps from negative to positive. This transition temperature can be called as the cross-over temperature $\left(T_{\mathrm{co}}\right)$. Equation $\mathrm{d} n_{\mathrm{o}} / \mathrm{d} T=0$ is solved to find out the value of this cross-over temperature.

\section{Results and discussion}

The clearing temperatures for two liquid crystal samples MLC-9200-000 and MLC-6608 are $362 \mathrm{~K}$ and $363 \mathrm{~K}$, respectively. The modified four-parameter model has four unknown constants $A, B, \Delta n_{\mathrm{o}}$, and $\beta$. These constants are obtained by fitting experimental data of $\sqrt{\left\langle n^{2}\right\rangle}$ and $\Delta n$. The fitting parameters for these two samples are listed in Table.

The results from measurements and continuous curves of modified four-parameter model for ordinary and extraordinary refractive indices are presented in Fig. 1. As the temperature increases, the extraordinary refractive index decreases sharply while the ordinary refractive index decreases initially but gradually increases near the clearing temperature. Behavior of $n_{\mathrm{e}}$ and $n_{\mathrm{o}}$ for MLC9200-000 and MLC-6608 is found to be similar as in the case of other organic liquids $[12,15,16]$. Figure 2 depicts the temperature dependence of experimental 
TABLE

Values of calculated fitting parameters for $\sqrt{\left\langle n^{2}\right\rangle}$ and $\Delta n$ of MLC-9200-000 and MLC-6608 at $\lambda=589 \mathrm{~nm}$.

\begin{tabular}{c|c|c|c|c}
\hline \hline \multirow{2}{*}{ LC sample } & \multicolumn{2}{|c|}{$\sqrt{\left\langle n^{2}\right\rangle}$} & \multicolumn{2}{|c}{$\Delta n$} \\
\cline { 2 - 5 } & $A$ & $B\left[\mathrm{~K}^{-1}\right]$ & $\Delta n_{\mathrm{o}}$ & $\beta$ \\
\hline MLC-9200-000 & 1.6307 & $-4.22 \times 10^{-4}$ & 0.1235 & 0.2288 \\
MLC-6608 & 1.6341 & $-4.41 \times 10^{-4}$ & 0.1177 & 0.2163
\end{tabular}

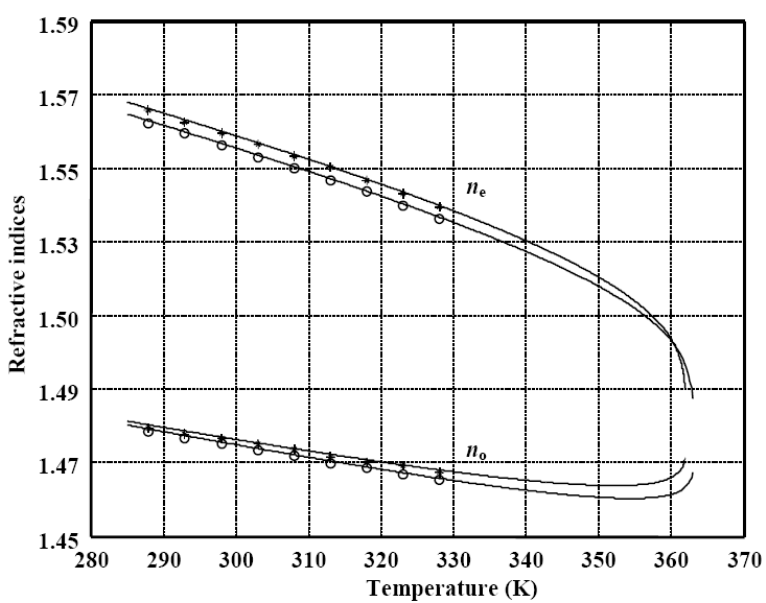

Fig. 1. Temperature dependence of refractive indices $\left(n_{\mathrm{e}}, n_{\mathrm{o}}\right)$. Stars $(*)$ represent the experimental data of $\left(n_{\mathrm{e}}, n_{\mathrm{o}}\right)$ for MLC-9200-000 and open circles (o) are the $\left(n_{\mathrm{e}}, n_{\mathrm{o}}\right)$ experimental data for MLC-6608. Solid lines are the fitting curves using four-parameter model.

results and theoretical curves of birefringence $\Delta n$. Birefringence decreases gradually as the operating temperature increases. A similar nature is found for both the LC samples. Near the clearing temperature, the two refractive indices are the same (i.e. $n_{\mathrm{e}}=n_{\mathrm{o}}$ ). Therefore, the birefringence becomes zero, when the temperature reaches up to a certain value equal to $T_{\mathrm{c}}$.

Usually, the birefringence is considered as a measure of order parameter in a liquid crystalline phase. The order parameter behaves in the same manner as the birefringence does. It decreases as the operating temperature increases and becomes zero as the temperature approaches $T_{\mathrm{c}}$. A comparison of order parameter values obtained in two different ways is presented in Fig. 3 for MLC-9200000 and in Fig. 4 for MLC-6608. These results are obtained using two methods: from birefringence measurements and Haller's extrapolation method. Zywucki and Kuczynski [12] have suggested that the results of order parameter determination using different procedures may differ. Here also, we find a different temperature dependence of order parameter. The difference between the results obtained by a 


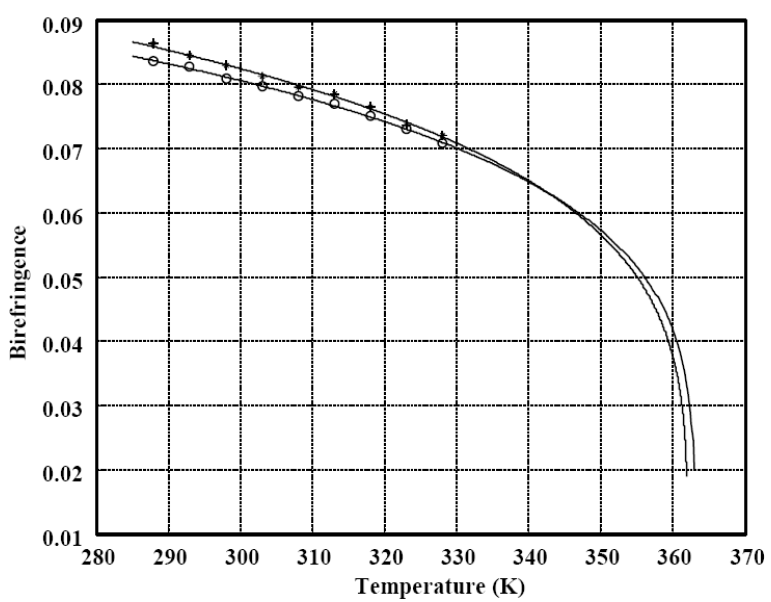

Fig. 2. Temperature dependence of birefringence $\Delta n$. Stars $(*)$ and open circles (o) are experimental data of $\Delta n$ for MLC-9200-000 and MLC-6608, respectively. Solid lines are the fitting curves using Eq. (4).

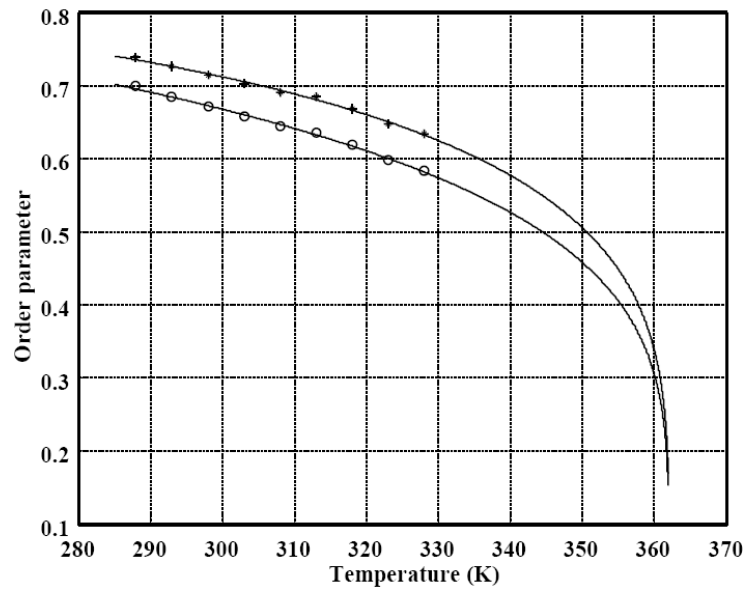

Fig. 3. Temperature dependence of order parameter $(Q, S)$ for MLC-9200-000. Stars (*) represent the experimental data of $Q$, using birefringence measurements. Open circles (o) are the experimental data of $S$, using Haller's extrapolation method. Solid lines are the fitting curves using Eqs. (9) and (10).

simple procedure based on birefringence measurements and using Haller's extrapolation method does not exvceed $\approx 4.0 \%$.

The variation of ratio of normalized polarizabilities $\alpha_{\mathrm{e}} / \alpha_{\mathrm{o}}$ with temperature has been plotted in Fig. 5. The ratio $\alpha_{\mathrm{e}} / \alpha_{\mathrm{o}}$ decreases with an increase in temperature. It is clear that when the temperature becomes equal to $T_{\mathrm{c}}$, the birefringence vanishes, i.e. both the refractive indices are no more different. Therefore, 


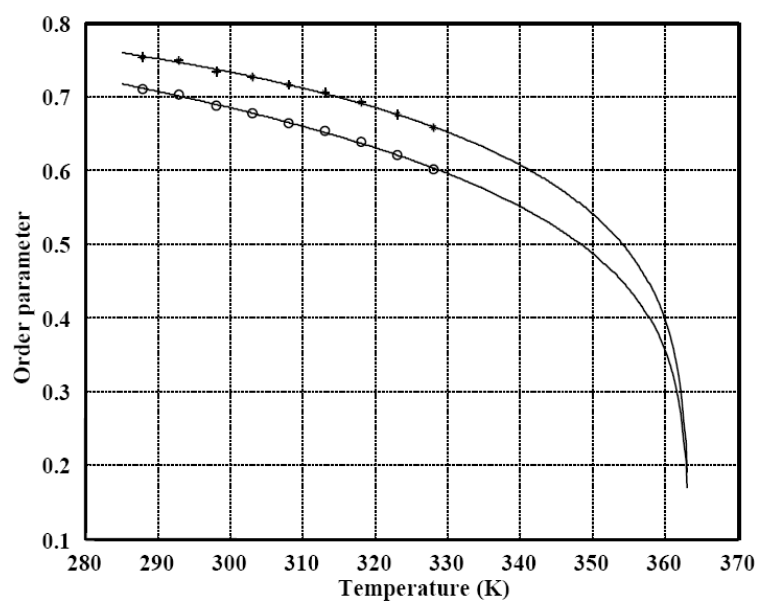

Fig. 4. Temperature dependence of order parameter $(Q, S)$ for MLC-6608. Stars (*) represent the experimental data of $Q$, using birefringence measurements. Open circles (०) are the experimental data of $S$, using Haller's extrapolation method. Solid lines are the fitting curves using Eqs. (9) and (10).

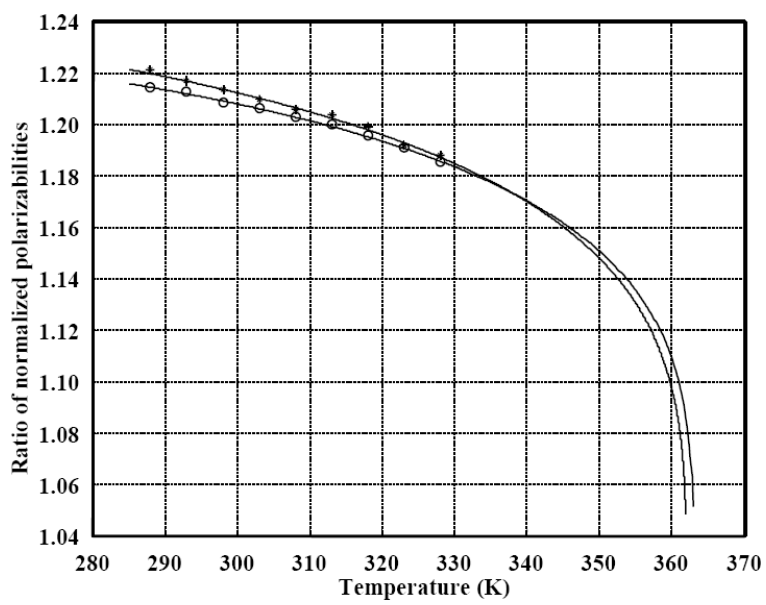

Fig. 5. Temperature dependence of the ratio of normalized polarizabilities $\alpha_{\mathrm{e}} / \alpha_{\mathrm{o}}$. Stars (*) represent the experimental data of $\alpha_{\mathrm{e}} / \alpha_{\mathrm{o}}$ for MLC-9200-000 and open circles (o) are the experimental data of $\alpha_{\mathrm{e}} / \alpha_{\mathrm{o}}$ for MLC-6608. Solid lines are the fitting curves using Eq. (12).

Eq. (12) confirms that near the clearing temperature the value of $\alpha_{\mathrm{e}} / \alpha_{\mathrm{o}}$ becomes one. The temperature dependence of $-\mathrm{d} n_{\mathrm{e}} / \mathrm{d} T$ and $\mathrm{d} n_{\mathrm{o}} / \mathrm{d} T$ is shown in Fig. 6 for MLC-9200-000 and MLC-6608. The calculated values of cross-over temperatures $\left(T_{\text {co }}\right)$ for MLC-9200-000 and MLC-6608 are 352.25 K and 354.44 K, respectively. The extraordinary refractive index decreases with increasing in temperature, there- 


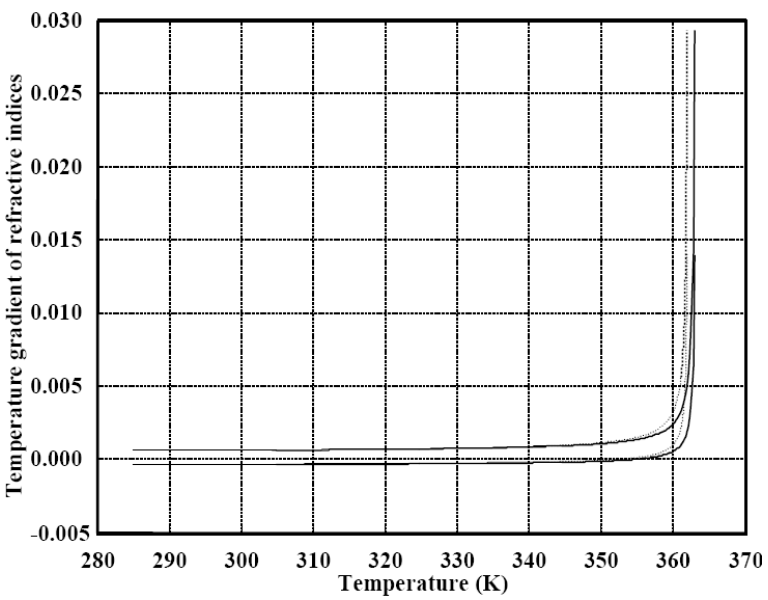

Fig. 6. Temperature dependence of refractive indices temperature gradients $\mathrm{d} n_{\mathrm{e}} / \mathrm{d} T$ and $\mathrm{d} n_{\mathrm{o}} / \mathrm{d} T$. Dashed lines are the fitting curves for MLC-9200-000 and solid lines are the fitting curves for MLC-6608 using Eq. (13A) and (13B).

fore its negative temperature gradient $\left(-\mathrm{d} n_{\mathrm{e}} / \mathrm{d} T\right)$ remains always positive for both the LC samples. However $n_{\mathrm{o}}$ decreases initially but near the clearing temperature it increases, so its temperature gradient $\mathrm{d} n_{\mathrm{o}} / \mathrm{d} T$ changes the sign from negative to positive value. The $\mathrm{d} n_{\mathrm{o}} / \mathrm{d} T$ is negative when the temperature is below $T_{\text {co }}$, whereas it becomes positive when the temperature is above $T_{\mathrm{co}}$.

\section{Conclusion}

The modified four-parameter model, explaining temperature dependence of liquid crystal refractive indices and different theoretical equations for other relevant parameters are used to check out the experimental results. Discrete experimental points and the fitting curves are plotted in the same graph for each parameter. This four-parameter model fits the experimental data very well. It can also be seen that all other experimental results are in good agreement with theory and the literature data. The fitting parameters are obtained by giving the linear fit to the experimental data of $\sqrt{\left\langle n^{2}\right\rangle}$ and $\Delta n$. Two methods for the determination of order parameter in liquid crystals are compared. The first method yields that the order parameter can be calculated directly from the macroscopic quantity the birefringence. Knowing the value of $\Delta n_{\mathrm{o}}$ (birefringence for crystalline state i.e., at $T=0 \mathrm{~K}$ ), the order parameter can be obtained from the ratio $\Delta n / \Delta n_{\mathrm{o}}$. Haller's extrapolation method is being used in the second approach for calculating the orientational order parameter. It is concluded that the order parameter computed from different procedures must not necessarily be identical. It has been found that $\mathrm{d} n_{\mathrm{e}} / \mathrm{d} T$ remains always negative. This means that $n_{\mathrm{e}}$ must decrease as temperature increases. However, $\mathrm{d} n_{\mathrm{o}} / \mathrm{d} T$ changes its sign near the cross-over tem- 
perature. This implies that $n_{\mathrm{o}}$ may decrease or increase depending on operating temperature.

\section{Acknowledgments}

The author wishes to acknowledge Mr. Ajeet Kumar, Department of Physics, Indian Institute of Technology, Roorkee, for the assistance provided by him to improve the manuscript.

\section{References}

[1] G.L. Ellis, B. Williamson, J. White, J. Emerg. Med. 7, 675 (1989).

[2] S. Sato, Opt. Rev. 6, 471 (1999).

[3] F. Li, N. Mukohzaka, N. Yoshida, Opt. Rev. 5, 174 (1998).

[4] W. Haase, F. Podgornov, J. Opt. Tech. 68, 677 (2001).

[5] A. Jakli, L.C. Chien, Liq. Cryst. 29, 377 (2002).

[6] S.T. Wu, Phys. Rev. A 33, 1270 (1986).

[7] S.T. Wu, C.S. Wu, M. Warenghem, M. Ismaili, Opt. Eng. 33, 1775 (1993).

[8] E.M. Averynov, J. Opt. Tech. 64, 471 (1997).

[9] J. Li, S. Gauza, S.T. Wu, J. Appl. Phys. 96, 19 (2004).

[10] T.N. Soorya, S. Gupta, A. Kumar, S. Jain, V.P. Arora, B. Bahadur, Ind. J. Pure Appl. Phys. 44, 524 (2006).

[11] J. Li, C.H. Wen, S. Gauza, R. Lu, S.T. Wu, IEEE/OSA J. Disp. Tech. 1, 51 (2005).

[12] B.J. Zywucki, W. Kuczynski, IEEE J. Trans. Dielec. Elec. Insl. 8, 512 (2001).

[13] I. Haller, Prog. Solid State Chem. 10, 103 (1975).

[14] M.F. Vuks, Opt. Spektrosk. 20, 644 (1966).

[15] A.K. Garg, V.K. Agarwal, B. Bahadur, Mol. Cryst. Liq. Cryst. 130, 11 (1985).

[16] R.K. Sarna, B. Bahadur, V.G. Bhide, Mol. Cryst. Liq. Cryst. 51, 117 (1979). 